\title{
Influence of Ulnar Translation of the Radial Shaft in Distal Radius Fracture on Distal Radioulnar Joint Instability
}

\author{
Hisao Moritomo, MD, $\mathrm{PhD}^{1}$ Shinsuke Omori, $\mathrm{MD}^{2}$ \\ ${ }^{1}$ Osaka Yukioka College of Health Science, Yukioka Hospital Hand \\ Center, Osaka, Japan \\ 2 Department of Orthopaedic Surgery, Osaka University Graduate \\ School of Medicine, Osaka, Japan
}

\begin{abstract}
Address for correspondence Hisao Moritomo, MD, PhD, Department of Physical Therapy, Osaka Yukioka college of Health Science, Yukioka Hospital Hand Center, 2-2-3, Ukita, Kita-ku, Osaka-shi, Osaka

530-0021, Japan (e-mail: moritomo@tcct.zaq.ne.jp).
\end{abstract}

J Wrist Surg 2014;3:18-21.
Abstract
Keywords
- distal oblique bundle
- interosseous membrane
- translation
- distal radius fracture
- distal radioulnar joint instability

It has been reported that the distal interosseous membrane (DIOM) of the forearm constrains the dorsal dislocation of the distal radius. A residual ulnar translation deformity of the radial shaft in distal radius fractures has the potential to cause distal radioulnar joint (DRUJ) instability when triangular fibrocartilage complex (TFCC) injury is also present, because it may result in detensioning of the DIOM. Correction of ulnar translation of the radial shaft is critical because it restores DIOM tension, which then firmly holds the ulnar head in the concavity of the sigmoid notch.
The distal interosseous membrane (DIOM) of the forearm, which is distal to the central band of the interosseous membrane, spans the radius and ulna at the dorsal region of the pronator quadratus muscle. The DIOM acts as a secondary soft tissue stabilizer of the distal radioulnar joint (DRUJ) when the triangular fibrocartilage complex (TFCC), which is the primary stabilizer of the DRUJ, is torn. ${ }^{1-4}$ The functional anatomy of the DIOM and an isometric thick fiber within the DIOM called the distal oblique bundle (DOB) has been reported. ${ }^{5-9}$ The DIOM and its effect on DRUJ instability in distal radius fractures have recently been attracting attention. $^{10-12}$

Distal radius fractures show various patterns of deformity. Ulnar translation of the proximal fragment, which is a reflection of the radial translation of the distal fragment, is one of the most common deformities; however, it is commonly overlooked or unrecognized. ${ }^{13}$ Ulnar translation of the proximal fragment has been described as a radial beak pattern, in which the radial part of the distal fragment creates the appearance of a large beak. ${ }^{14}$ This pattern has been seen in $\sim 50 \%$ of distal radius fractures, and it correlates with the anatomical location of the insertion of the brachioradialis distal tendon. ${ }^{14}$
A residual ulnar translation deformity of the proximal radial shaft has the potential to cause DRUJ instability when a TFCC injury is also present, because it may result in detensioning of the DIOM ${ }^{11}$ ( $\mathbf{- F i g . 1}$ ). However, to the best of our knowledge, the influence of an ulnar translation deformity of the proximal radial shaft on DRUJ stability has never been quantitatively investigated.

\section{Preliminary Biomechanical Experiment}

We performed a preliminary biomechanical experiment to investigate the influence of ulnar translation of the proximal fragment on DRUJ instability. We used three fresh-frozen cadaveric upper limbs, all of which had a distinct DOB in the DIOM. All specimens were amputated above the elbow. Skin, tendons, joint capsules, and muscles were removed, sparing the ligaments, and interosseous membranes. The TFCC, the extensor carpi ulnaris tendon subsheath, and extensor retinaculum were also preserved. Then, the humerus and ulna were fixed to the testing apparatus with the elbow in $90^{\circ}$ flexion, which allowed the radius to rotate freely around the ulna. Both the radiocarpal and midcarpal joints were fixed in the neutral wrist position using two Kirschner wires (K-wires) of 3.0-mm diameter.
Copyright $\odot 2014$ by Thieme Medical Publishers, Inc., 333 Seventh Avenue, New York, NY 10001, USA. Tel: +1(212) 584-4662.
DOI http://dx.doi.org/ 10.1055/s-0033-1364093. ISSN 2163-3916. 


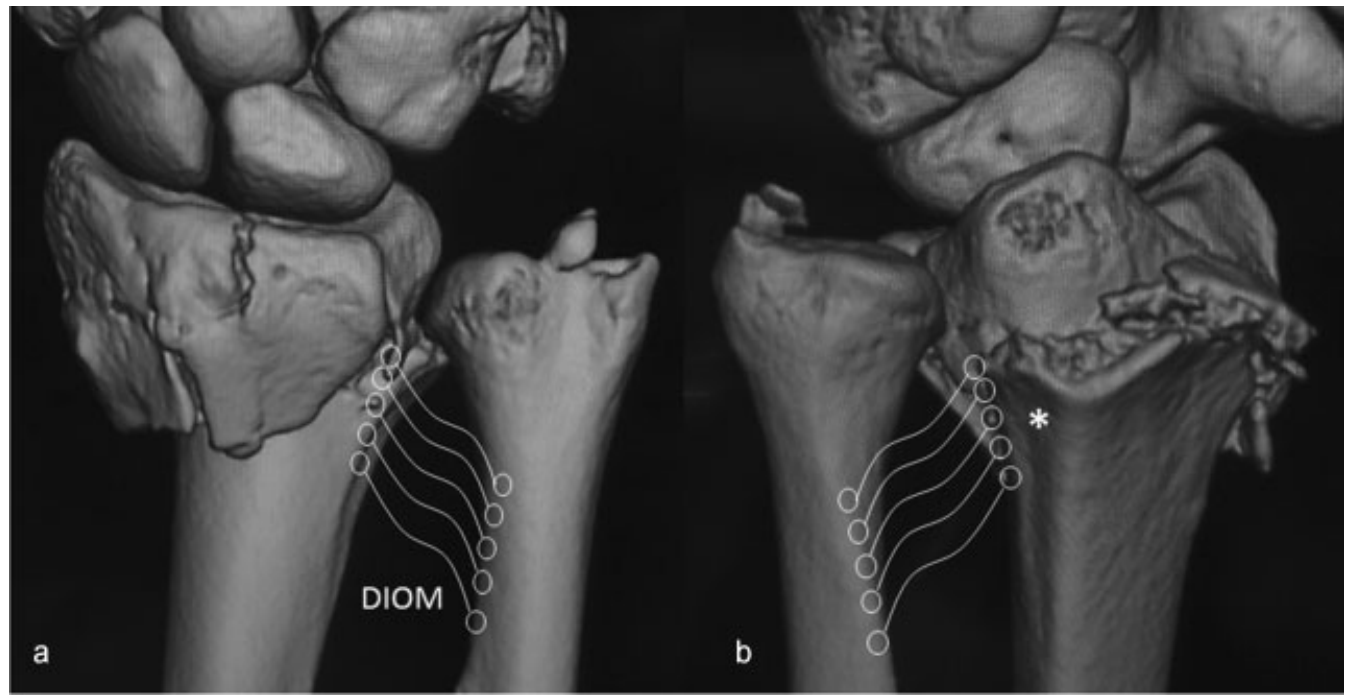

Fig. 1 Three-dimensional computed tomographic images of a common distal radius fracture with TFCC and the extensor carpi ulnaris tendon sheath rupture (Orbay's Type B fracture) demonstrating ulnar translation deformity of the radial shaft and detensioning of the distal interosseous membrane (DIOM). The radial attachment of DIOM is located at the dorsal region of the medial wall of the radius at which the deep portion of the pronator quadratus muscle inserts $\left({ }^{*}\right)$. (a) Dorsal and (b) palmar views of the right wrist.

A 3-dimensional space electromagnetic tracking device (3SPACE FASTRAK; Polhemus, Colchester, Vermont, USA) was used to measure the DRUJ mobility. One sensor was placed on the ulnar aspect of the distal ulna, and another was placed on the radial aspect of the distal radius. A screw of $4-\mathrm{mm}$ diameter was inserted into the ulnar corner of the distal radius to apply loading in the dorsal and palmar directions. Passive DRUJ mobility was tested by translating the distal radius relative to the ulna in a dorsopalmar direction with a load of $19.6 \mathrm{~N}$ using a weight in a neutral forearm rotation. The magnitudes of displacement were measured under various conditions, including the following: intact TFCC and DIOM ( - Fig. 2a), complete sectioning of the TFCC including the foveal fibers of the radioulnar ligaments with an intact DIOM (-Fig. 2b), additional 5-mm ulnar translation of the radial shaft at the level of the proximal margin of the sigmoid notch with an external fixator (-Fig. 2c), and complete sectioning of DIOM after reducing the ulnar translation deformity. As a result, under the condition in which the TFCC was completely sectioned with an intact DIOM, the radius dislocated palmarly, resulting in dorsal translation of the ulnar head relative to the radius but not absolutely. Bidirectional (both palmar and dorsal) dislocation of the radius occurred after additional ulnar translation of the radial shaft. The bidirectional dislocation was corrected simply by reducing the ulnar translation and was reproduced by DIOM sectioning after reducing the deformity. These results suggested the following: (1) The intact DIOM constrained dorsal dislocation of the radius but did not prevent palmar dislocation; (2) a residual 5-mm ulnar translation deformity of the proximal radius detensioned the DIOM, resulting in bidirectional (palmar and dorsal) DRUJ instability when a TFCC

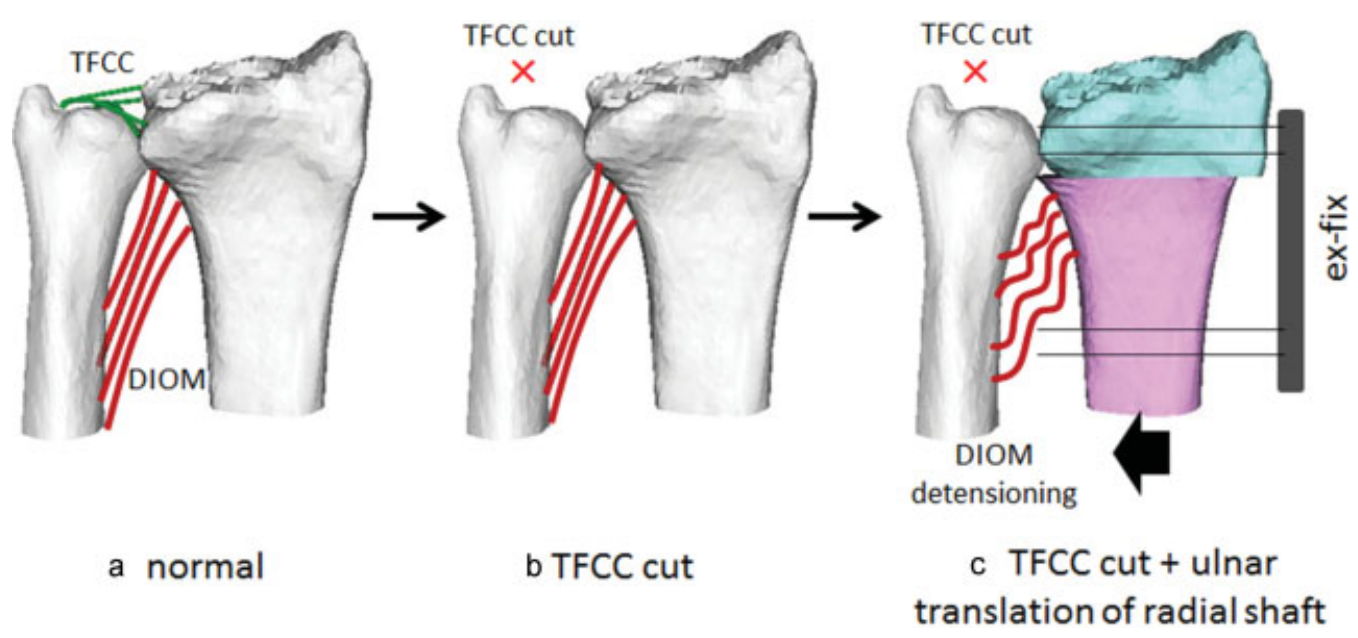

Fig. 2 Three conditions of soft tissue stabilizers of DRUJ. (a) Intact TFCC and DIOM. (b) Complete sectioning of TFCC with intact DIOM. (c) Additional 5-mm ulnar translation of the radial shaft at the level of the proximal margin of the sigmoid notch using an external fixator. 
injury was also present; and (3) when the TFCC and DIOM were cut after anatomic reduction of the skeletal structures, bidirectional DRUJ instability persisted.

\section{Discussion}

Previous cadaveric biomechanical studies have revealed that the DIOM plays an important role in constraining the dorsal dislocation of the radius at the DRUJ; however, it seldom constrains the palmar dislocation of the radius. ${ }^{1-4}$ We confirmed in our study that the radius did not dislocate dorsally as long as the DIOM remained intact when the TFCC was completely sectioned. However, this important function of the DIOM in maintaining DRUJ stability in the face of a distal radius fracture has not been well recognized.

The DIOM predominantly constrains the dorsal dislocation of the radius because of its anatomic features. The radial attachment of the DIOM spreads from the dorsal rim of the sigmoid notch (distally) to the ulnar aspect of the radial shaft (proximally) ${ }^{8}$ (-Fig. 3a). The radial attachment of the DOB, the thickest fiber of the DIOM, is located at the most dorsal region of the medial wall of the radius at which the deep portion of the pronator quadratus muscle inserts (-Fig. 1b). The DOB is stretched between the proximal margin of the dorsal rim of the sigmoid notch and the most radial aspect of the ulnar shaft $4-5 \mathrm{~cm}$ proximal to the distal end of the ulna. In the axial view, the DOB passes from the dorsoradial side to the ulnopalmar side ( - Fig. 3b). The DOB perhaps becomes taut and works effectively in wrist supination in which the radius shifts dorsally, thereby compressing the ulnar head on the palmar rim of the sigmoid notch due to the dorsal orientation of the radial attachment of the DOB.

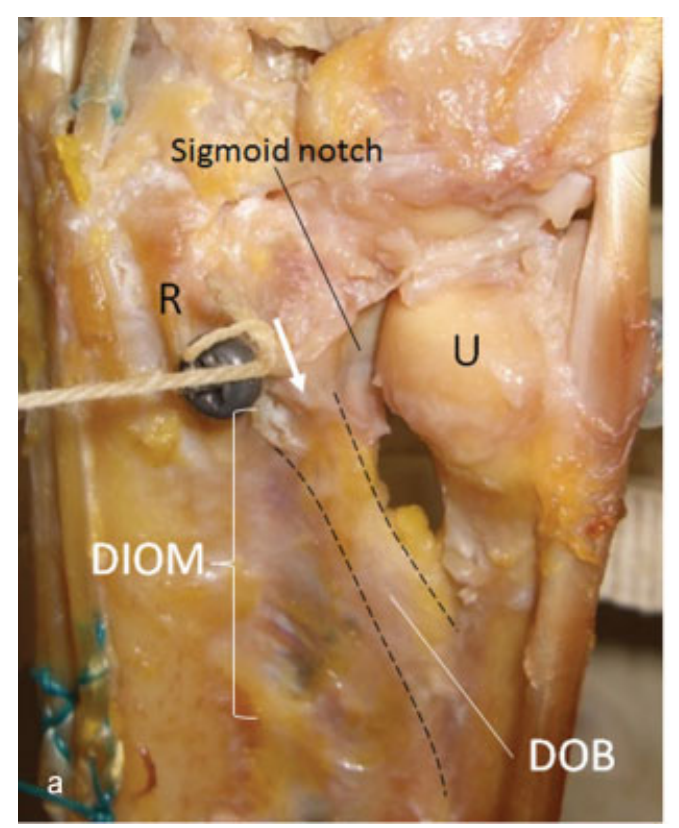

Orbay has classified ulnar-side injuries that are concomitant with the distal radius fractures into three types. ${ }^{12}$ In type A, the TFCC and DIOM remain intact; they need no specific treatment other than the restoration of the bony anatomy. In type B, the TFCC and the extensor carpi ulnaris tendon sheath rupture, but the DIOM remains intact. DRUJ subluxation is corrected, and adequate stability is restored after the anatomic reduction of the skeletal structures. In type C, the TFCC, the extensor carpi ulnaris tendon sheath, and the DIOM, including the DOB, all rupture. Therefore, all the ligamentous support for the DRUJ is lost. After anatomic reduction of the skeletal structures, either subluxation of DRUJ persists or clinical testing shows DRUJ instability. The persistent DRUJ instability needs specific intervention such as TFCC reconstruction.

In cases where the TFCC is severely injured and there is detensioning of the DIOM due to ulnar translation of the radial shaft, the DRUJ gap increases, allowing dorsal and palmar dislocation of the sigmoid notch of the radius relative to the ulnar head. The results of our experiment suggest that, if ulnar translation deformities of the radial shaft in type B fractures are not adequately corrected, type B fractures become similar to type $\mathrm{C}$ fractures and DRUJ instability persists.

Therefore, we should be careful when correcting deformities in distal radius fractures, especially Orbay's type B fractures. During volar plate fixation, residual ulnar translation of the radial shaft can easily be corrected, as suggested by Ross. ${ }^{11}$ The single screw that is present in the sliding hole is slightly loosened but maintained in place; it forms a pivot point for the correction. A claw-type bone holder can then be positioned with one limb over the ulnar border of the radius just proximal to the fracture line and one limb over the radial side of the plate or through one of the holes on the plate. When the clamp is tightened, progressive ulnar translation of

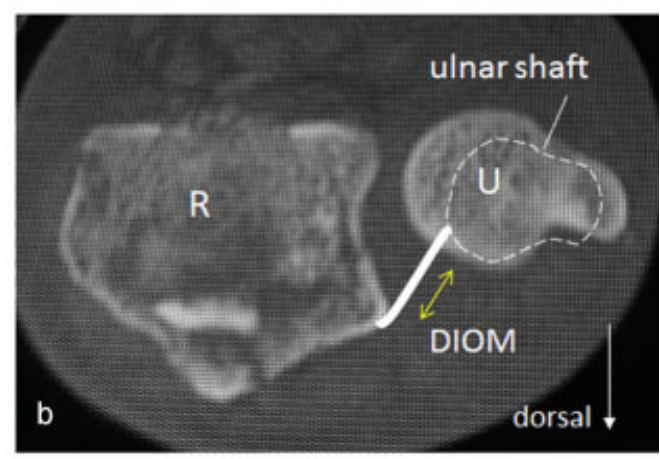

Fig. 3 (a) The distal oblique bundle (DOB), the thickest fiber of the DIOM, is stretched between the proximal margin of the dorsal rim of the sigmoid notch and the most radial aspect of the ulnar shaft $4-5 \mathrm{~cm}$ proximal to the distal end of the ulna. (b) In the axial view, DOB passes from the dorsoradial side to the ulnopalmar side. 
the distal fragment occurs with the proximal screw acting as a pivot point. When satisfactory translation has been achieved, the single screw is tightened, and as long as length has also been restored, the remaining shaft screws can be inserted.

Dorsal dislocation of the distal radius is constrained by the intact DIOM when the TFCC is ruptured. In case of a TFCC injury, additional ulnar translation of the radial shaft or DIOM rupture causes dorsal and palmar instability of DRUJ. Correction of ulnar translation of the radial shaft restores the DIOM tension, which subsequently holds the ulnar head firmly in the concavity of the sigmoid notch. Thus, hand surgeons need to recognize the function of the DIOM with injuries in which DRUJ instability accompanies distal radius fractures.

\section{Conflict of Interest}

None

\section{References}

1 Kihara H, Short WH, Werner FW, Fortino MD, Palmer AK. The stabilizing mechanism of the distal radioulnar joint during pronation and supination. J Hand Surg Am 1995;20(6):930-936

2 Ward LD, Ambrose CG, Masson MV, Levaro F. The role of the distal radioulnar ligaments, interosseous membrane, and joint capsule in distal radioulnar joint stability. J Hand Surg Am 2000;25(2): 341-351

3 Stuart PR, Berger RA, Linscheid RL, An KN. The dorsopalmar stability of the distal radioulnar joint. J Hand Surg Am 2000; 25(4):689-699
4 Watanabe H, Berger RA, Berglund LJ, Zobitz ME, An KN. Contribution of the interosseous membrane to distal radioulnar joint constraint. J Hand Surg Am 2005;30(6):1164-1171

5 Noda K, Goto A, Murase T, Sugamoto K, Yoshikawa H, Moritomo H. Interosseous membrane of the forearm: an anatomical study of ligament attachment locations. J Hand Surg Am 2009;34(3): 415-422

6 Moritomo H, Noda K, Goto A, Murase T, Yoshikawa H, Sugamoto K. Interosseous membrane of the forearm: length change of ligaments during forearm rotation. J Hand Surg Am 2009;34(4):685-691

7 Kitamura T, Moritomo H, Arimitsu S, et al. The biomechanical effect of the distal interosseous membrane on distal radioulnar joint stability: a preliminary anatomic study. J Hand Surg Am 2011;36(10):1626-1630

8 Moritomo $\mathrm{H}$. The distal oblique bundle of the distal interosseous membrane of the forearm. J Wrist Surg 2013;2(1):93-94

9 Omori S, Moritomo H, Murase T, et al. Changes in length of the radioulnar ligament and distal oblique bundle after Colles' fracture. J Plast Surg Hand Surg 2013;47(5):409-414

10 Jupiter JB. Commentary: the effect of ulnar styloid fractures on patient-rated outcomes after volar locking plating of distal radius fractures. J Hand Surg Am 2009;34(9):1603-1604

11 Ross M. Volar angle stable plating for distal radius fractures. In: Slutsky DJ, ed. Principles and Practice of Wrist Surgery. Philadelphia, PA: WB Saunders; 2010:133-136

12 Orbay JL. Ulnar head and styloid fractures. In: Slutsky DJ, ed. Principles and Practice of Wrist Surgery. Philadelphia, PA: Saunders; 2010:198-204

13 Rapley JH, Kearny JP, Schrayer A, Viegas SF. Ulnar translation, a commonly overlooked, unrecognized deformity of distal radius fractures: techniques to correct the malalignment. Tech Hand Up Extrem Surg 2008;12(3):166-169

14 Koh S, Andersen CR, Buford WL Jr, Patterson RM, Viegas SF. Anatomy of the distal brachioradialis and its potential relationship to distal radius fracture. J Hand Surg Am 2006;31(1):2-8 